\title{
Experiments With Multi-Modal Interfaces in a Context-Aware City Guide
}

\author{
Christian Bornträger ${ }^{1,2}$, Keith Cheverst ${ }^{2}$, Nigel Davies ${ }^{2}$, Alan Dix ${ }^{2}$, \\ Adrian Friday ${ }^{2}$, and Jochen Seitz ${ }^{1}$ \\ 1 Technische Universität Ilmenau, \\ Fakultät für Elektrotechnik und Informationstechnik \\ 98693 Ilmenau, Germany \\ 2 Lancaster University, \\ Computing Department, \\ Bailrigg, Lancaster LA1 4YR, UK
}

\begin{abstract}
In recent years there has been considerable research into the development of mobile context-aware applications. The canonical example of such an application is the context-aware tour-guide that offers city visitors information tailored to their preferences and environment. The nature of the user interface for these applications is critical to their success. Moreover, the user interface and the nature and modality of information presented to the user impacts on many aspects of the system's overall requirements, such as screen size and network provision. Current prototypes have used a range of different interfaces developed in a largely ad-hoc fashion and there has been no systematic exploration of user preferences for information modality in mobile context-aware applications. In this paper we describe a series of experiments with multi-modal interfaces for context-aware city guides. The experiments build on our earlier research into the GUIDE system and include a series of field trials involving members of the general public. We report on the results of these experiments and extract design guidelines for the developers of future mobile context-aware applications.
\end{abstract}

\section{Introduction}

In recent years there has been significant research interest in developing mobile context-aware applications. Such applications typically provide information and services that are tailored to the user's context, including their environment, preferences and usage history. Probably the most familiar example of these forms of application is the 'context-aware tour-guide', of which a number of prototype systems have been developed (e.g. [1, 8]). Among these systems, one of the most widely reported is the Lancaster GUIDE system developed by the authors $[8,5]$.

GUIDE provides users with a comprehensive mobile context-aware tour-guide that can be used to explore the city of Lancaster. The system is based on a tablet PC (the Fujitsu TeamPad) that was made available to the general public from the Lancaster Tourist Information Centre. GUIDE uses a familiar webbrowser based user interface to present users with a mixture of hand crafted and 
generated textual descriptions, pictures and maps about key attractions in the city. In addition to this information, GUIDE also provides a number of additional features including guided walking tours, group messaging facilities and simple interactive booking services.

The initial GUIDE system, developed and deployed in the late 1990s, used solely static media types that were requested by the user explicitly (i.e. we adopted a PULL-based approach to information dissemination [7]). The decision to restrict ourselves to static media types (i.e. text and images) was taken for two reasons. Firstly, the hardware platform lacked the capability to deal with audio and video playback. Secondly, and more importantly, feedback from users and tourism officers had suggested that audio was not an appropriate media type for use in a city environment. Specifically, there were concerns that users would not wish to use headphones because they would feel isolated from their environment (including other members of their party) and would not wish to use a loudspeaker for fear of attracting attention to themselves (and hence feeling even more conspicuous than a tourist normally does). The decision not to use audio was at odds with the prevailing commercial trend for indoor guide systems, which often employ 'walkman' style cassette players or handheld solid state units - but reflected the more comprehensive visual capabilities of our end-system and the different deployment environment.

In later tests [6] the GUIDE system was extended with additional capabilities including audio. In these tests we compared the PULL-based approach used in the first version of GUIDE with an information PUSH model. Though there are different ways of defining PUSH and PULL, our definition is based on the model of Cheverst et al. [7]: information PULL is characterised by the fact that the user expects the information (i.e. in response to some explicit action) while in information PUSH, the user receives the information unexpectedly (e.g. in response to some contextual trigger).

In early 2002 the decision was taken to begin a total redesign of the GUIDE system allowing us to explore a new set of research challenges in the field of mobile context-aware computing. As part of this redesign process we became interested in understanding which media types are best suited for providing information on city attractions. This is clearly important since the range of media types that needs to be supported impacts all aspects of a system's design, including the choice of end-system, interaction method and required level of network, processor and storage support. Our study of the literature indicated that while different prototype tour-guide systems had used different interfaces, these had largely been developed on an ad-hoc basis. Furthermore, none of the widely deployed systems had implemented multiple user interfaces and attempted to systematically study user preferences with regard to these interfaces. As a result, there are no design guidelines available to indicate which media types and interface modalities are most appropriate for providing information to users of mobile context-aware tour-guides. In this paper we aim to provide such guidelines based on a series of user trials. 
In section 2 we describe an experimental mobile context-aware tour-guide application that we developed specifically to research user interface modality issues. Our experimental methodology is described in section 3 and our results in section 4 . These results include quantitative data we obtained from log files as well as qualitative results from interviews with users. Section 5 provides an interpretation of the data and explains why some results met our expectations and why some did not. The observations of the experimenter are used to round up the analysis. Section 6 gives an overview of related work and section 7 contains our concluding remarks.

\section{Experimental Application}

To support our experiments into multi-modal user interfaces we developed a new tour-guide application for the city of Lancaster. This prototype is based on a Compaq iPAQ PocketPC, which compared to the existing GUIDE units, provides support for a wider range of media types, a smaller form factor, an improved (i.e. colour) display and a more up-to-date image (recent interviews with tourists indicated that the Fujitsu TeamPad looked somewhat old-fashioned when compared to the new PocketPCs).

Our system allows tourists to receive information about city attractions in the form of text, pictures or audio commentary, supplemented with a map available in various styles and resolutions. Figure 1 shows screenshots of all of the visual interfaces. These interfaces fill the complete screen, as experiments with

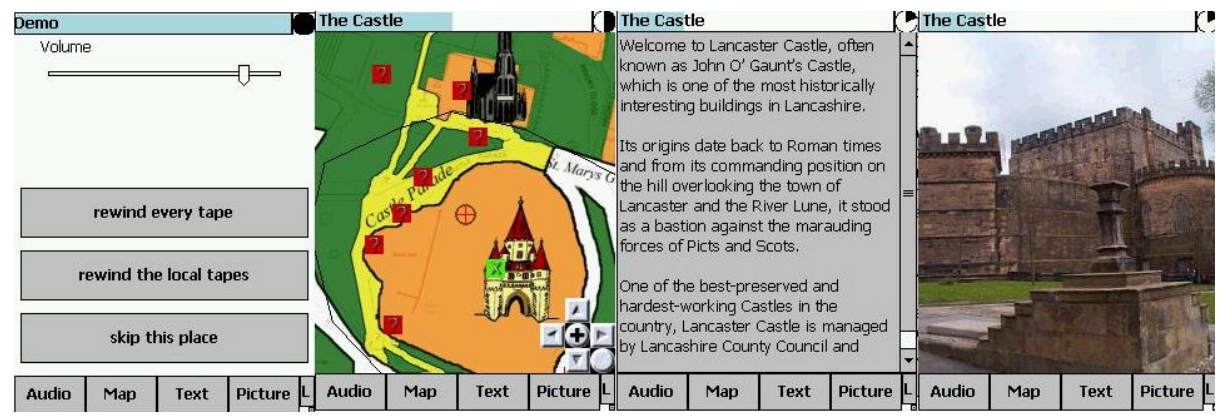

Fig. 1. There are 4 visual interfaces besides the audio

combinations of visual interfaces failed since it was not possible to achieve good readability (because the resolution and screen size, 240 by 320 pixels, is too small). Reading from left to right, the interfaces are as follows:

Audio Control This interface provides users with a simple means of controlling the audio playback of the device. 
Map This interface provides users with a scrollable, resizable map of the area, overlayed with markers indicating tourist attractions and an icon representing the user's current location.

Text A description of the current tourist attraction in textual form. This text is identical to the audio commentary.

Picture A picture of the current tourist attraction.

Users switch between the interfaces using the tabs at the bottom of each screen. While only one visual interface can be shown at a time, the audio is always available with all visual interfaces. The audio can be played through the built-in speaker or via headphones, and can be muted if not required. Because of the limited quality of speech synthesis, the output is constructed from prerecorded mp3-files that were spoken by a native English speaker. It is hoped that this has avoided any negative feedback associated with speech synthesis that might have influenced the results.

In addition to the variant shown in the screenshot, the map was made available in different resolutions and styles. The map offers two different graphical representations of the available information "hot spots", i.e. areas for which information is available. The first representation uses a translucent blue colour that covers the areas of the map where attractions are located. The second representation of hot spots uses red question marks that turn into green 'X's after the information has been played. Users can switch between these representations by clicking on the appropriate button at the bottom of the screen.

The information flow used in this prototype is PUSH-based. For each attraction we define a surrounding geographic area equivalent to the attraction's nimbus [2] or "hot spot". When a user enters this area we push the information associated with the attraction to the user. The size and shape of these geographical areas were determined by trial and error. We used a standard GPS receiver attached to the iPAQ both to define the hot spot regions and to track the tourists' position during the trials (this represents another departure from the original GUIDE system, which obtained location information from its point of attachment to the network).

The descriptions of attractions are composed in a hierarchy: the logic of the system ensured that the general information about the attraction is always provided before more detailed information is given. For example, before the prototype played information about the "Shire Hall", some general information was first provided about the "Lancaster Castle".

The following example text is an extract (about one third) of a typical description:

"Around the arch of the Shire Hall is perhaps the most famous display of heraldry in the country. This display, numbering over 600 shields, includes those of the monarchs, the High Sheriffs of Lancashire, and the Constables of the Castle. The High Sheriffs date back to the $12^{t h}$ Century. This is an ancient rank with responsibility for organising the administration of justice..." 
A key part of the prototype was the logic of replacement [6]: in a PUSH-based system it is always hard to tell when and how to update information since there is a risk of overwriting data that is still being used. Our prototype uses a time based solution. More specifically, if a tourist leaves the nimbus of the currently active attraction but the audio is still playing, the device waits for 10 seconds. If the tourist does not return to the attraction within this period and enters the nimbus of another attraction, the current presentation is stopped and the device changes to the new attraction. The device also offers the user a manual override that allows navigation backwards and forwards using the left and right buttons of the iPAQ. This control can be used to rewind, if the prototype replaces information the tourist was still listening to.

Since in this experiment we were principally interested in exploring the use of multi-modal interfaces and not in developing another version of GUIDE, the context stimuli used to tailor information was less than that used in the original system. Specifically, only the usage history and the location was used to tailor the presentation rather than including factors such as user interests and the time of day.

The system was designed to provide comprehensive usage data for later analysis. Every interaction, including interface changes and button presses are logged. This information is recorded with the current time, the current GPS coordinates and the currently active attraction. The data is saved into a log file that can be used to replay the complete test run. The log files are parsed afterwards by a specially designed program to gather statistics.

\section{Experimental Methodology}

Our tests were conducted during November and December 2002 in an area known as "Castle Hill" in Lancaster, UK. This area covers Lancaster Castle and its immediate surroundings and was chosen because it is close to the city's Tourist Information Centre and rich in tourist attractions. Figure 2 shows a map of the test site. In total there were 16 separate tourist attractions all located within approximately 5 minutes walk of the castle. The area is predominately pedestrianised with a low volume of traffic (on those roads that allow vehicles).

Most of the test subjects were recruited from visitors to the Tourist Information Centre. Basing ourselves within this council-run facility lent a degree of respectability to the test and helped avoid tourists thinking that we were trying to sell them something. In order to convince people to participate in the test we advertised the trial as a free opportunity to learn more about Lancaster. We accepted tourists with no knowledge of Lancaster as well as those that had studied the city before their trip.

After recruiting a tourist or tourist group ${ }^{3}$, we started the test with a short introduction to the device. This introduction was designed to last about 3 minutes: our previous experiences have led us to believe that minimising the time necessary to learn how to use a system is critical in the tourism domain, since users

\footnotetext{
${ }^{3}$ We define a tourist group as being two or more persons co-visiting the city.
} 


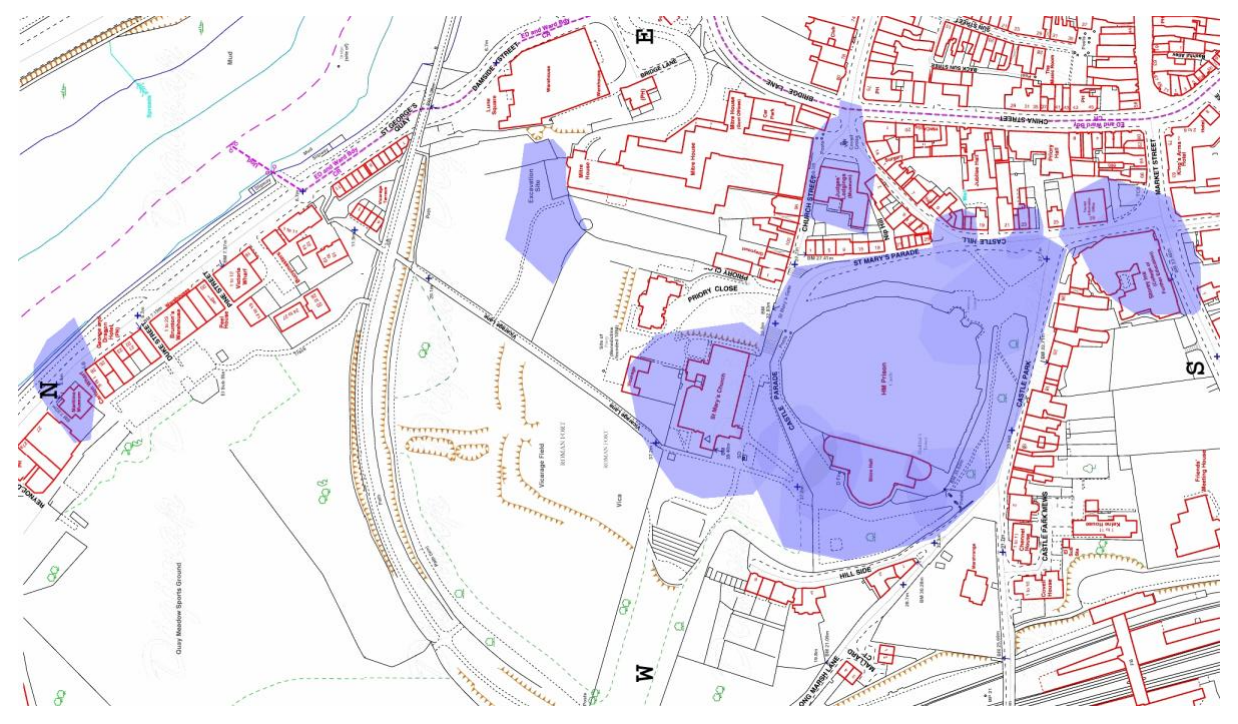

Fig. 2. The hot spots around Lancaster castle

perceive this learning process as cutting into their leisure time. To make the introduction as efficient as possible, the prototype offers a tutorial mode, which behaves much like the normal mode and offers all possible interactions. However, instead of giving information about attractions, the tutorial mode has short descriptions of the interfaces. In our tests the experimenter introduced the tourists to each interface, showed the possible interactions, and described the purposes of the various buttons. The tourist could then play with the device and ask questions until they felt comfortable with its operation, at which point the guide unit was changed from tutorial mode into normal mode.

Finally, the general task we set the tourists was to discover and explore the area and use the interfaces to maximise the experience. We did not provide them with any further information on the purpose of the experiment. The unit was left with the tourist to use as they wished. An experimenter followed at a distance of 5 to 10 metres to help in a case of a problem and to observe patterns of behaviour. The intention was not to control the test, rather to observe, though we accept that this process may have affected how some users interacted with the system. We set no time limit for the tourists, they could use the system for as long as they liked.

Once the tourists had finished using the prototype we asked questions in a semi-structured interview. This interview focused on the users preferences for different interfaces. Specifically we asked whether users preferred the audio or textual information and why this was the case. Details were also asked about the map, the picture and the text interfaces. For example, we asked how important these interfaces were to the user and what they were used for. If users indicated that a particular interface was poor or little used, we asked why and described 
alternative designs to gain an understanding of the factors that affected user reactions. In addition to interface selection, we were also interested to learn how users reacted to the PUSH model used in the prototype. For example, do people have problems if only a PUSH-based interface is available? As tourists normally do not understand the terms PULL and PUSH, the methods were described as "having a list of attractions and choose the appropriate one" and "getting the information without asking for it". Finally, we asked for further comments on what additional features the tourist would like to have and how they would rate the GUIDE system overall. If the participants agreed, the interview was recorded for later analysis.

\section{Results}

During our field trial we conducted 20 independent tests, 11 with individuals and 9 with groups of two or more tourists. In 4 cases the test runs were particularly short as the participants were in a hurry. As the period of interaction in these cases was extremely short, we chose to disregard this data and focus on the remaining 9 individuals and 7 groups. The groups were composed of 5 sets of couples, one group of 3 and one of 4 persons. The age of the youngest person involved in the trial was 19 and the oldest was 71.

The average time spent using the system was 11 minutes with a maximum of 21 minutes $^{4}$. Most tests ended prematurely as the subjects wished to continue their tour inside a particular attraction. Overall, most test participants were quite enthusiastic about the prototype system.

\subsection{User Interaction}

All user interactions with the system were logged. We analysed the log data to determine which of the available user interface modalities was preferred. The results show that there is no single user interface preference valid across all of the test subjects. However, it is possible to partition the results into 3 separate groups: the majority of participants - 11 out of 16 - spent most of the time using the map interface. 4 of the 16 preferred the picture view and 1 individual used the textual descriptions most of the time. Table 1 shows a summary of the test data. The result of the modality choice is more obvious: nobody used the volume control to mute the audio and hence these interfaces were always used in conjunction with the audio commentary.

Effect of group size. One aspect we were interested in was whether the group size affected the choice of user interface. Though there is a small observable difference in the time spent in a given interface by groups versus individuals, the results are not significant according to a t-test (the error-probability is 20 percent).

\footnotetext{
${ }^{4}$ The total amount of audio that is available around Lancaster Castle is 13 minutes, if played continuously.
} 


\begin{tabular}{|c|c|c|c|}
\hline & All Participants & Groups & Individuals \\
\hline \hline Audiocontrol & 0 & 0 & 0 \\
Map & 11 & 4 & 7 \\
Text & 1 & 0 & 1 \\
Picture & 4 & 3 & 1 \\
\hline
\end{tabular}

Table 1. Distribution of test persons according to the most used interface

Effect of mobility. Another important aspect in our analysis is the mobility of the user. Figure 3 shows the change in use according to the speed of the tourist (as measured by the GPS compass).

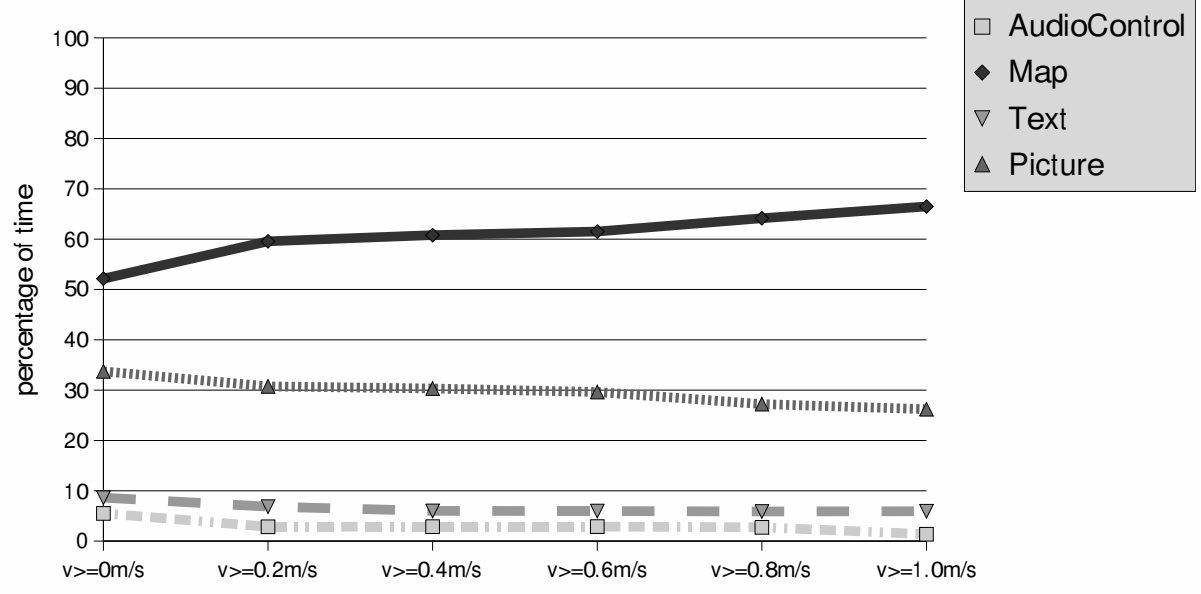

Fig. 3. The speed influence on the interface choice

For most participants there is a correlation between the subject's speed and map usage: the higher the speed the more the map is used. This is valid for both the map-oriented subjects as well as the picture-oriented subjects. The picture appears to be used less while moving. A t-test comparing a slow and a fast speed indicates with less than 5 percent error that the change in the usage of the map and picture is statistically significant.

Effect of audio. We have observed a difference between the use of the interface modalities depending on whether audio is being used. Figure 4 shows the average time spent in an interface while (a) the prototype is playing and (b) the prototype is not playing audio.

While the audio is being played the usage of the picture view increases significantly. Conversely, the map view is preferred when the audio commentary is not played. 

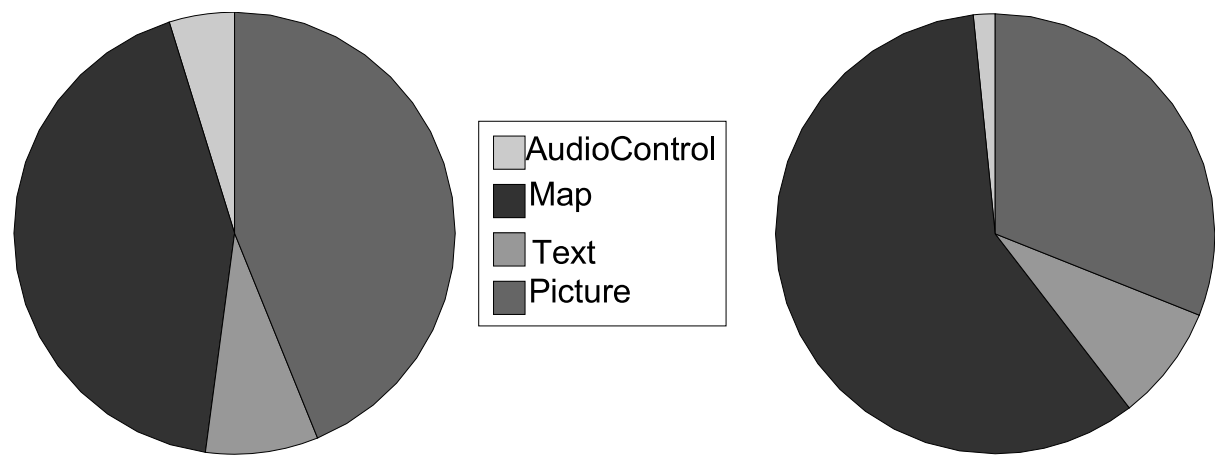

$\begin{array}{ll}\text { (a) audio is playing } & \text { (b) no audio is playing }\end{array}$

Fig. 4. The influence of audio

\subsection{User Interviews}

We found a number of interesting results from the semi-structured interviews. Most of the tourists thought that audio guides were preferable to text based guides. The main reason given was that it is easier to follow the information when you are able to look at the attractions while they are being described.

Importance of text. Asked how important the additional text was, most subjects stated that they did not need the text. We then suggested some possible changes to the system, such as highlighting the currently spoken text, reducing or increasing the amount of information. The vast majority of the subjects stated that it would not matter. The availability of headlines or keywords instead of whole passages of text was also rejected by the majority of people. About one third thought that keywords might be of some use. During the test a small number of subjects said that the text was useful to replay information they'd heard in the commentary.

Importance of the map. Asked which interface the users considered to be the most important, most people stated that the map view was essential. Even if precise audio guidance was available, such as "mind the step" or "turn right on the next corner into Church Street", people stated that they would still like to have a map. Typical comments made by the tourists included "I want to see what's available", "people are visual" as well as "I always have to ask twice if I only have spoken guidance". In contrast, one subject stated "I got lost on maps".

We asked people about the design of the maps. We asked whether the subjects believed that the map should use a compass and automatically compensate for the direction of the unit. There was no common answer. All possible opinions from "absolutely necessary" and "I hate it if I have to do it on my own", to "might be useful" and "it would distract me" were given. Most people agreed on the use of the map - it was used to find out "where to go". 
Importance of the picture. In our tests, the picture was mainly used for "seeing if I am right' or "finding the object the device is talking about". The subjects universally agreed that a picture of an attraction was really helpful.

Importance of how information is delivered. Some subjects mentioned that it would be nice to have the ability to request information: they wanted to know if it was worth going somewhere in advance. Although they were quite happy with the prototype itself they missed the level of control of a user initiated (PULL-based) approach.

One tourist complained about the information replacement strategy. Though there was a back button, he was surprised that the device started to talk about a different attraction while he was walking. He suggested that the device should give a warning signal or other hint. (Design guideline: In a case of replacing information a warning should be included, no matter how clever the replacement logic is.) Possibilities for such a warning might include a mobile phone like 'reception bar' or reducing the volume of the audio. Alternatively the colour of the current object on the map might be faded out or the title bar could show "Leaving...".

Delivery of audio information. We offered all single tourists new headphones and earphones: everyone rejected the offer. One reason given was "headphones look strange". Another was that tourists did not want to look like tourists. Ironically, the use of an audio guide which delivers audio information out loud clearly demarks the user as a tourist. One possible explanation might be that headphones are visible over a long distance, whereas the audio can only be heard over a relatively short distance. Only one person switched to headphones: he was hard of hearing and unable to understand the speech under normal conditions.

\section{Analysis}

In some cases the lessons learnt from our testing and observations could have been predicted. The increased use of the map while moving is consistent with our expectations, as maps are well known and familiar tools for navigation. Similarly, the text interface is extremely difficult to use while moving due to the level of attention it demands - especially when preoccupied with other cognitive tasks such as avoiding traffic and other pedestrians. Furthermore, a subject is unlikely to want to look at a picture of the last attraction while moving away from it. These factors all help to explain why the map is the dominant form of interaction while on the move.

Audio and Movement. As stated in the results section above, we have observed an influence of the audio on the choice of secondary media type. There may also be a correlation between user mobility and the audio: quite often the user stopped soon after the audio started playing. From the perspective of the experimenter there is a typical set of observable behaviour. As soon as the audio starts to play, the test subject stopped and looked around. If they were not successful in determining the attraction mentioned in the commentary, they interacted with the device and looked around again. Normally the tourist needed 
some time to locate the unknown attraction, especially if it was not immediately obvious. (Design guideline: in a PUSH-based audio system the designer should plan to include some time for the user to locate the attraction. Consequently, no important facts should be pushed during the first few seconds of audio.) This observation of the experimenter is supported by the results of the interviews. The test subjects used the map for finding where to go and the picture for identifying the attraction. This behaviour is also clearly common sense.

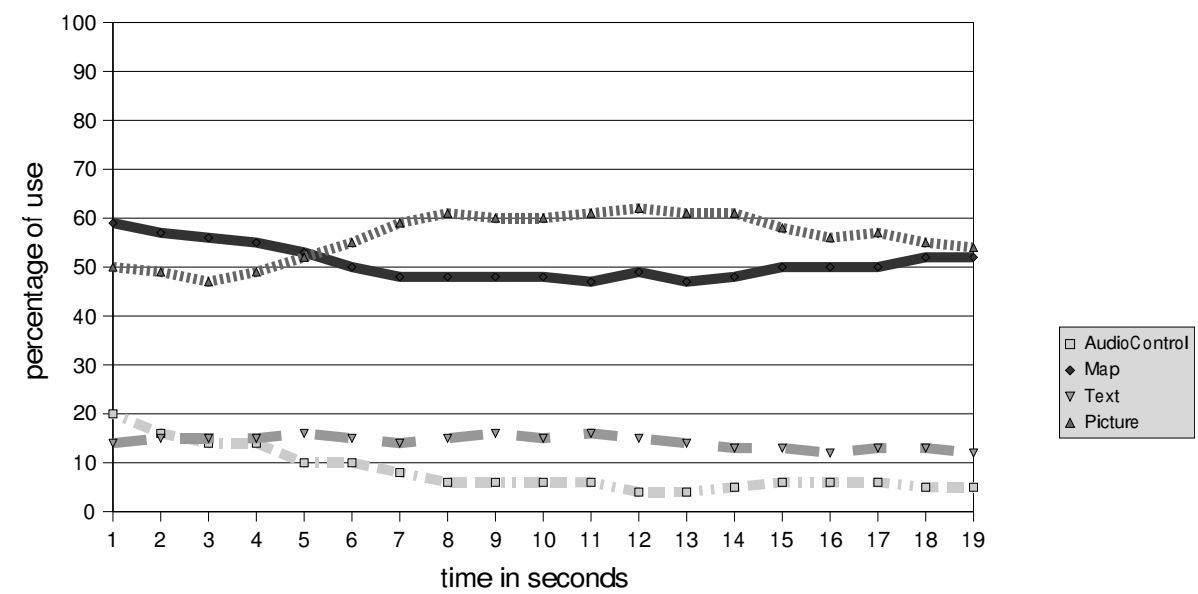

Fig. 5. First seconds after the audio started

Control of Audio. Figure 5 shows the percentage that each modality is in use during the first few seconds after the audio begins. From these results we can see that after approximately 5 seconds the principal choice of interface switches from map to picture - further supporting the anecdotal observation of the experimenter. The results also highlight that the audio controls are often used during these first few seconds. Our interpretation of this phenomenon is that the audio control interface allows the subject to restart the audio. A representation of a typical usage cycle is shown in figure 6 .

Acceptance of Audio. As we have already mentioned, one of the prime justifications for the success of an audio based guide is the ability of the user to be able to be looking at the sites while simultaneously being presented with accompanying information. Similar results have been identified in indoor guide systems [21]: the attention of the user has to be split between the guide, the attraction and their companions. Text guides are seen to be particularly problematic for groups as they are difficult to use collaboratively - in contrast, with an audio guide it is much easier to share the experience. Such sharing might even promote interaction within the group. 


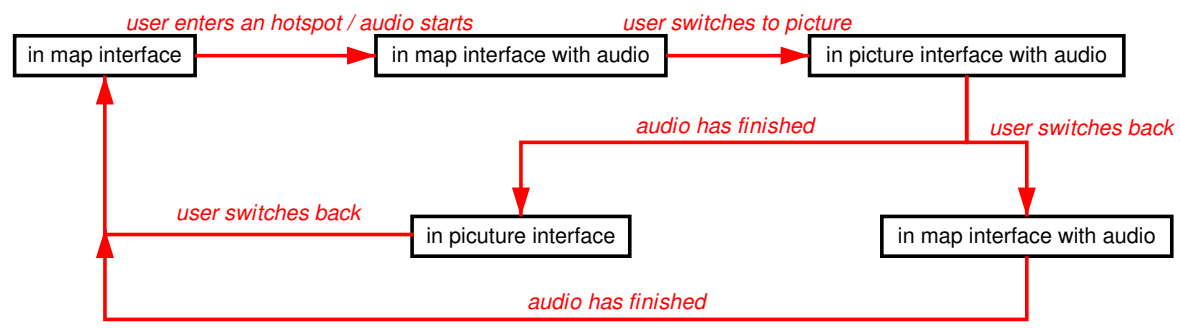

Fig. 6. The typical interaction cycle of a PUSH-based audio guide

Our experiments also revealed that a guide system definitely should offer more than just the audio commentary: both the map and the picture were considered as necessary in our interviews. (Design guideline: a guide system should offer an optimised interface for navigation and an optimised interface for identification.)

Effect of Group Size. We were surprised to find that being part of a group was not statistically significant. Though groups show a small tendency towards using more pictures and less text, the statistical analysis reports this as being within the margin of error. One possible explanation for this outcome might be that most groups had a "leader". This person was the principal user of the device and typically decided where to go - using the device like a single person.

In the small sample set and group sizes we studied, everyone was able to hear the audio acceptably. The audio was found to trigger an interaction with the other group members: when the audio started and the group leader could not find the attraction immediately, the companions were asked for help. In other cases the audio seemed adequate for the other group members as the leader would decide where to go and normally point to the attraction.

In our largest group (with 4 members), the behaviour was different: as the audio began the leader had to call everybody together, he then rewound the audio once they had assembled. Consequently, this group used the audio control the most frequently. Clearly, no definite conclusions can be drawn from a single statistical sample.

Use of Headphones. The most surprising result was the universal avoidance of headphones. One possible explanation was given by the tourists - headphones look strange. Tourists do not want to be easily recognised as tourists. This seems somewhat anomalous given the popularity of personal music players and handsfree kits for mobile phones. Curiously, one of our subjects was already wearing a 'Walkman' with his own set of headphones. He rejected the offer to use his headphones with the iPAQ, insisting that he used the speaker instead. One possible explanation might be the wish to avoid feeling isolated from the environment. The use of open headphones instead of semi-open or closed ones might help to reduce this effect. (Design guideline: headphones might be unwanted. An alternative audio system should be available.) Other possible explanations might be the fear of invasion of personal privacy [11] or fear of the unknown. Audio that 
is pushed to the user without their specific interaction might be deemed as an invasion of personal privacy - this effect might be further enhanced by the intimate form of delivery (through headphones). A user might also fear the lack of control over the spontaneity or volume, given that the audio is played directly into the ear. We suspect that these results are especially interesting as a number of other projects have proposed the use of spatialised audio with headphones for tourist guide applications [9,10]. A further possibility for future work would be to determine if the reluctance to use headphones is only valid for outdoor guides.

\section{Related Work}

There have been numerous research projects involving context-aware tourist guide systems. Besides the Lancaster GUIDE the most well known is the Cyberguide project at Georgia Tech [1]. The Cyberguide project was created in the early 1990s and was one of the first context-aware applications. The system was developed using Apple Newtons and relied on a combination of text and images to provide information to visitors.

The Lancaster GUIDE project has previously experimented with audio. An extended version of the GUIDE application running on an Fujitsu TeamPad was used. The test [6] showed that tourists happily accepted a GUIDE version with additional audio-PUSH. Our tests confirmed this result even in the absence of any PULL mechanism, though some users stated that they would have liked to have had the option of requesting additional information.

The HIPS project [3] created a prototype tour-guide system called Hippie [17] designed for use in museums. Hippie can be personalised and the information is web-based. Interestingly, tourists can start interacting with Hippie at home and continue in the museum. Hippie appears to offer most of the functionality of a web based system and hence can include multiple media types.

Other projects dealing with audio interfaces are AudioGPS [10], Guided By Voices [13], Hear \& There [20], Audio Aura [16], LISTEN [9], and Nomadic Radio [18]. These projects use audio as the main interface or are audio only. Nomadic Radio, AudioGPS, LISTEN, and Hear \& There use spatial audio for transmitting information. As our tests showed a reluctance against headphones at least for outdoor guides, alternative designs should be considered. Apart from Hear \& There none of the mentioned projects offer an additional visual interface. This might be a problematic design as tourist in our tests stated that they like to have a visual component as well. Nevertheless, audio only systems have the advantage of being useful for blind people. Projects that deal with guidance in unknown areas are Strider [14, 4, 15], Personal Guidance System [12] and InfraVoice [19]. Strider uses an audio map describing the way and Personal Guidance System use an acoustic display for giving information about distance and angle. InfraVoice is based on cheap receivers and a pre-installed network of infrared-transmitters in a town. These transmitters send directed infrared beams containing information. The user can find these object by pointing the receiver to tune into the information. All of these projects have to live without any possibility of visual 
interaction, and consequently a lot of effort has had to be made in promoting their usability. The huge effort necessary in supporting navigation in such systems, confirms the results of this test: an appropriate interface for navigation is mandatory. Visual components like maps are a common choice which are also well known to potential users.

\section{Conclusion}

As mobile context-aware applications begin to proliferate it is important there are design guidelines available to help developers. The canonical example of a mobile context-aware application is a tour-guide system and yet, despite the development of numerous prototype systems, there has to date been no systematic study of user acceptance of different user interface modalities in such systems. In this paper we have presented the results of our efforts to address this shortcoming.

Our experiments have highlighted a number of issues that we believe are generally applicable to mobile context-aware tour-guide systems. Firstly, users clearly benefit from having information available in multiple modalities. Moreover, different users exploit this information in different ways, making it difficult to make a strong case for the inclusion or omission of a specific form of information. Secondly, users were clearly able to make use of different interfaces for navigation and for information access, implying that it might be possible to develop a system with different interfaces optimised for these distinct tasks. Thirdly, we observe that when audio is pushed to users they typically do not give it their full attention for the first few seconds, implying that important information should not be provided at this time. Fourthly, we observe that no matter how clear the replacement logic appears to be for PUSH-based information delivery, confirmation or explanation of this action is almost always desirable. Finally, we note that while audio is becoming a more accepted form of information delivery, users exhibit a remarkable reluctance to wear headphones. This is a surprise to us given the popularity of personal music players and hands-free kits for mobile phones.

In conclusion, we believe that our experiments provide a useful set of guidelines for developers of future mobile context-aware tour-guides. The extent to which these guidelines can be applied to other context-aware application domains is a subject for further study.

\section{References}

1. Gregory Abowd, Christopher Atkeson, Jason Hong, Sue Long, Rob Kooper, and Mike Pinkerton. Cyberguide: A Mobile Context-Aware Tour Guide. ACM Wireless Networks, 3:421-433, 1997.

2. Steve Benford and Lennart E. Fahlen. A Spatial Model of Interaction in Large Virtual Environments. In Proceedings of The Third European Conference on Computer Supported Cooperative Work, pages 109-124, 1993. 
3. Jonathan Broadbent and Patrizia Marti. Location Aware Mobile Interactive Guides: Usability Issues. In Proceedings of The Fourth International Conference on Hypermedia and Interactivity in Museums, pages 88-98, 1997.

4. Michael Busboom and Michael May. Mobile Navigation for the Blind. In Proceedings of The Wearable Computer Conference (ICWC), 1999.

5. Keith Cheverst, Nigel Davies, Keith Mitchell, Adrian Friday, and Christos Efstratiou. Developing a context-aware electronic tourist guide: some issues and experiences. In Proceedings of CHI'00, pages 17-24, 2000.

6. Keith Cheverst, Keith Mitchell, and Nigel Davies. Exploring Context-aware Information Push. Personal and Ubiquitous Computing, 6:276-281, 2002.

7. Keith Cheverst and Gareth Smith. Exploring the notion of information push and pull with respect to the user intention and disruption. In Proceedings of The International workshop on Distributed and Disappearing User Interfaces in Ubiquitous Computing, pages 67-72, November 2001.

8. N. Davies, K. Mitchell, K. Cheverst, and G. Blair. Developing a Context Sensitive Tourist Guide. In Proceedings of The First Workshop on Human Computer Interaction for Mobile Devices, 1998.

9. Gerhard Eckel. Immersive Audio-Augmented Environments. In Proceedings of the 8th Biennial Symposium on Arts and Technology, 2001.

10. Simon Holland and David R. Morse. Audio GPS: spatial audio in a minimal attention interface. In Proceedings of The Third International Workshop on Human Computer Interaction with Mobile Devices, 2001.

11. Marc Langheinrich. Privacy by design - principles of privacy-aware ubiquitous systems. In G.D. Abowd, B. Brumitt, and S. Shafer, editors, Ubicomp 2001 Proceedings, volume 2201 of Lecture Notes in Computer Science, pages 273-291. Springer, 2001.

12. Jack M. Loomis, Reginald G. Golledge, and Roberta L. Klatzky. Navigation system for the blind: Auditory display modes and guidance. Presence: Teleoperators and Virtual Environments, 7(2):193-203, 1998.

13. K. Lyons, M. Gandy, and T. Starner. Guided by Voices: An Audio Augmented Reality System. In Proceedings of The International Conference on Auditory Display, 2000.

14. Michael May. Strider: A Presentation. In Proceedings of The Wearable Computer Conference (ICWC), 1998.

15. Michael May. Accessible GPS Navigation and Digital Map Information for Blind Consumers. In Proceedings of The Wearable Computer Conference (ICWC), 2000.

16. Elizabeth D. Mynatt, Maribeth Back, Roy Want, Michael Baer, and Jason B. Ellis. Designing Audio Aura. In Proceedings of The Conference on Human Factors in Computing Systems, pages 566-573, 1998.

17. R. Oppermann, M. Specht, and I. Jaceniak. Hippie: A Nomadic Information System. Lecture Notes in Computer Science, 1707:330-, 1999.

18. Reinhard Oppermann and Marcus Specht. A nomadic information system for adaptive exhibition guidance. In ICHIM, pages 103-109, 1999.

19. Royal National Institute for the Blind. InfraVoice. http://www.rnib.org.uk/jmu/infravoice.htm.

20. Joseph Rozier, Karrie Karahalios, and Judith Donath. Hear \& There: An Augmented Reality System of Linked Audio. In Proceedings of The International Conference on Auditory Display, 2000.

21. Allison Woodruff, Paul M. Aoki, Amy Hurst, and Margaret H. Szymanski. Electronic Guidebooks and Visitor Attention. In Proceedings of The International Conference on Hypermedia and Interactivity in Museums, pages 437-454, 2001. 